\title{
A Comparison of Personality Characteristics and Psychiatric Symptomatology between Upper Airway Resistance Syndrome and Obstructive Sleep Apnea Syndrome
}

\author{
Soo-Jung So ${ }^{1}$, Heon-Jeong Lee ${ }^{\circledR}$, Seung-Gul Kang ${ }^{2}$, Chul-Hyun Cho ${ }^{1}$, Ho-Kyoung Yoon ${ }^{1}$, and Leen Kim ${ }^{1}$ \\ ${ }^{1}$ Department of Psychiatry, Korea University College of Medicine, Seoul, Republic of Korea \\ ${ }^{2}$ Department of Psychiatry, Gil Medical Center, Gachon Medical School, Incheon, Republic of Korea
}

\begin{abstract}
Objective To investigate the personality characteristics of patients with upper airway resistance syndrome (UARS) and those of patients with obstructive sleep apnea syndrome (OSAS).

Methods Eighty-eight patients with UARS and 365 patients with OSAS participated. All patients had a diagnostic full-night attended polysomnography (PSG) and completed the Athens Insomnia Scale (AIS), Pittsburgh Sleep Quality Index (PSQI), Epworth Sleepiness Scale (ESS), Symptom Checklist-90-Revision (SCL-90-R) and Eysenck Personality Questionnaire (EPQ).

Results The UARS group scored significantly higher than the OSAS group on the ESS, AIS, and PSQI ( $<<0.001)$. The scores of all SCL-90-R subscales in the UARS group were significantly higher than those in the OSA group (all $\mathrm{p}<0.001$, except for somatization, $\mathrm{p}=0.016)$. Patients with UARS scored lower on EPQ-E (extroversion/introversion) $(\mathrm{p}=0.006)$ and EPQ-L (lie) ( $<<0.001)$ than those with OSA. UARS patients also showed higher scores on EPQ-P (psychoticism) $(\mathrm{p}=0.002)$ and EPQ-N (neuroticism) $(\mathrm{p}<0.001)$ than OSAS patients.

Conclusion Our results suggest that patients with UARS have worse subjective sleep quality than OSAS patients in spite of their better PSG findings. UARS patients tend to have more neurotic and sensitive personalities than patients with OSAS, which may be a cause of the clinical features of UARS.

Psychiatry Investig 2015;12(2):183-189
\end{abstract}

Key Words Upper airway resistance syndrome, Obstructive sleep apnea, Personality characteristics.

\section{INTRODUCTION}

Upper airway resistance syndrome (UARS) is a sleep disorder characterized by complaints of excessive daytime sleepiness and frequent arousals during sleep preceded by increased respiratory efforts without overt apneas. Since a report on UARS was published in 1993 by Guilleminault et al. ${ }^{1}$ several studies on the clinical features of this syndrome have been reported. ${ }^{2-4}$ Nevertheless, the question of whether UARS is a distinct disease or a prodrome of obstructive sleep apnea syndrome (OSAS) is still a matter of debate.

Received: June 10, 2014 Revised: August 4, 2014

Accepted: August 24, 2014 Available online: February 2, 2015

$\triangle$ Correspondence: Heon-Jeong Lee, $\mathrm{MD}, \mathrm{PhD}$

Department of Psychiatry, Anam Hospital, Korea University College of Medicine, 73 Inchon-ro, Seongbuk-gu, Seoul 136-705, Republic of Korea Tel: +82-2-920-5815, Fax: +82-2-928-6706, E-mail: leehjeong@korea.ac.kr

(a) This is an Open Access article distributed under the terms of the Creative Commons Attribution Non-Commercial License (http://creativecommons.org/licenses/bync/3.0) which permits unrestricted non-commercial use, distribution, and reproduction in any medium, provided the original work is properly cited.
Some studies have investigated differences between patients with UARS and patients with OSAS. An early study found that patients with UARS complain more frequently of chronic insomnia and daytime sleepiness or fatigue than patients with OSAS, although respiratory abnormalities are more severe in OSAS patients than UARS patients. ${ }^{5}$ Other studies suggested that patients with UARS and OSAS differ in their presenting symptoms; the symptoms of UARS closely resemble those of the functional somatic syndromes. ${ }^{4,6}$ In addition, evidence suggests that patients with UARS are more impaired in their daily functioning and psychomotor performance than patients with OSAS.?

A few studies have suggested that patients with UARS have personal characteristics that differ from patients with OSAS. Guilleminault and colleagues suggested that patients with UARS have an increased parasympathetic component compared with OSAS patients. ${ }^{8,9}$ They also reported that the arousal threshold is lower in UARS, and this causes patients to wake up in response to small increases in respiratory effort. ${ }^{10,11}$ 
These findings have led us to hypothesize that patients with UARS have different personality characteristics from patients with OSAS. Many researchers have observed symptoms of depression and anxiety associated sleep-disordered breathing. ${ }^{12-14}$ Psychiatric assessments of patients with OSAS have also been reported, ${ }^{15-17}$ and patients with OSAS may have not only depression, but also psychopathological symptoms. ${ }^{16}$ However, no study has included psychiatric assessment of patients with UARS. Thus, the present study objectives were to compare personality characteristics and psychiatric symptomatology of UARS patients with those of OSAS patients and to explore personality characteristics as a possible cause of UARS.

\section{METHODS}

\section{Subjects}

All patients underwent laboratory-based full-night attended polysomnography (PSG) at the Sleep Disorder Center of Korea University Hospital, Seoul, South Korea. The patients were referred for suspected sleep-disordered breathing, insomnia, or hypersomnia. All cases were full-night diagnostic studies and excluded continuous positive airway pressure titration and split-night studies. A total of 883 diagnostic overnight sleep studies between January 2008 and September 2013 were evaluated. Among them, cases of restless legs syndrome/periodic limb movement disorders, narcolepsy, REM sleep behavior disorder and other sleep disorders according to the International Classification of Sleep Disorders, 2nd Edition (ICSD-2) ${ }^{18}$ were excluded based on NPSG recording and clinical evaluation. Patients with comorbidity of major psychiatric disorders [presence of Axis I diagnoses according to the Diagnostic and Statistical Manual of Mental Disorders, 4th Edition (DSMIV) ${ }^{19}$ and mental retardation], neurological disorders including stroke or head injury, and major medical disorders or other conditions that could influence on psychiatric and personality evaluation were excluded. Cases with incomplete data for the personality and psychiatric evaluations were also excluded from the study. This study was approved by the Ethics Committee of Korea University Medical Center.

\section{Psychiatric measurements}

All patients completed a sleep questionnaire that included the Athens Insomnia Scale (AIS), Pittsburgh Sleep Quality Index (PSQI), and Epworth Sleepiness Scale (ESS). The Athens Insomnia Scale (AIS) is a self-assessment psychometric instrument designed for quantifying sleep difficulty based on the ICD-10 criteria. ${ }^{20}$ It consists of eight items: the first five pertain to sleep induction, awakenings during the night, final awakening, total sleep duration, and sleep quality, while the last three concern well-being, functioning capacity, and sleepiness dur- ing the day. Both the entire eight-item scale (AIS-8) and the brief five-item version (AIS-5), which contains only the first five items, were utilized in this study.

The Pittsburgh Sleep Quality Index (PSQI) is a self-rated questionnaire that assesses sleep quality and disturbances over a 1-month time period. ${ }^{21}$ Nineteen individual items generate seven component scores: subjective sleep quality, sleep latency, sleep duration, habitual sleep efficiency, sleep disturbances, use of sleeping medication, and daytime dysfunction. The sum of scores for these seven components yields one global score.

The Epworth Sleepiness Scale (ESS) is a simple method for measuring the general level of daytime sleepiness or sleep propensity in adults. ${ }^{22,23}$ The ESS is a brief, self-administered questionnaire that asks the subject to rate on a four-point scale ( 0 3) his or her chances of dozing in each of eight specific situations that are commonly met in daily life $(0=$ would never doze; $3=$ high chance of dozing). The ESS score is the sum of the eight item-scores and can range from 0 to 24 . It provides a measurement of the subject's average sleep propensity in daily life.

The subjects' psychiatric symptoms were measured using Symptom Checklist-90-Revision (SCL-90-R), which is a multidimensional, self-report symptom inventory developed by Derogatis et al. ${ }^{24,25}$ The SCL-90-R consists of 90 items in total, which are divided into 9 symptom dimensions: somatization, obsessive-compulsive, interpersonal sensitivity, depression, anxiety, hostility, phobic anxiety, paranoid ideation, and psychoticism. The study subjects were asked to select from " $0=$ no problem" to " $4=$ very serious" to describe the extent of their symptoms.

For assessment of personality traits, we used the Eysenck Personality Questionnaire (EPQ) in this study, specifically the Korean-Version EPQ standardized by Lee and Eysenck. ${ }^{26}$ The EPQ contains four-item measures of psychoticism (social-psychopath, solitary, troublesome, cruel, and inhumane traits), neuroticism (anxious, worrying, moody and frequently depressed), extraversion (sociable, craves excitement, carefree, and optimistic), and lie (social desirability). ${ }^{27,28}$

\section{Evaluation of sleep-disordered breathing}

PSG was performed using a montage of 4-lead electroencephalography (EEG), electrocardiography, 2-lead electro-oculography, submental electromyography (EMG), bilateral anterior tibialis EMG, fingertip pulse oximetry, snore sensor, nasal and oral airflow, and chest and abdominal movement; airflow through the nose was measured with a nasal pressure transducer and thermistor. All parameters were recorded with Somnologica software and Embla S700/A10 (Embla System, Broomfield, CO, USA) and were scored initially by a certified technician and then reviewed by two doctors. Sleep architecture was scored in 30-s epochs, and sleep staging was per- 
formed according to the recommended AASM guidelines. ${ }^{29}$ Respiratory effort-related arousal (RERA) were defined as a sequence of breaths lasting at least 10 seconds characterized by increasing respiratory effort or flattening of the nasal pressure waveform leading to arousal when it does not meet the criteria for apnea or hyponea. In this study, the clinical diagnosis of UARS was established by 1) an apnea/hypopnea index (AHI) of $<10 / \mathrm{h} ; 2$ ) demonstration of flow limitation and increased respiratory efforts with arousal (RERA index $\geq \mathrm{AHI}$ ); and 3) the existence of excessive daytime somnolence (ESS $\geq 7$ ). The diagnosis of OSAS was established by an AHI $\geq 10 / \mathrm{h}$.

Although there is some controversy on the diagnostic criteria for UARS, we consider an AHI of $<10 / \mathrm{hr}$ as a criterion of UARS in accordance with previous studies. ${ }^{4}$ One reason we applied this criterion is that Asians exhibit more craniofacial bony restriction known to induce OSAS. ${ }^{30-35}$ When OSAS is defined by an $\mathrm{AHI}>5$, its prevalence in Korea is estimated too high ( $27 \%$ and $16 \%$ in men and women, respectively), according to a previous study. ${ }^{36}$

In terms of ESS score criteria, we also apply a low cut-off score of 7. Given cultural differences in lifestyle, inaccuracy of the ESS based on evidence in previous studies has been suggested. ${ }^{37,38}$ The number of people who drive themselves in automobiles is much smaller in Korea than in Western countries, especially among women and the elderly. Furthermore, the floor living lifestyle in Korea and Japan is different from the lifestyle of sitting in a chair in China and Western countries. Korean people who have floor living lifestyles usually think "sitting" in the questionnaire means squatting or kneeling as well as sitting in a chair. Because dozing when squatting or kneeling is quite rare, ESS scores tend to be lower than expect- ed in Korea. Therefore, we set the score of 7 as a cut-off score.

\section{Statistical analysis}

Statistical analyses were performed with SPSS version 18.0 (SPSS Inc., Chicago, IL, USA). Differences in group characteristics were analyzed with independent t-tests. To control for the effect of potentially confounding variables, such as age and sex, multivariate analysis of covariance (MANCOVA) was used. The mean scores of sleep questionnaires and psychological inventories were compared across the two diagnostic groups using MANCOVA. The score of each item of the SCL90-R and EPQ were the dependent variables, and age and sex were the covariates used in the MANCOVA. Test results were considered significant when $\mathrm{p}<0.05$.

\section{RESULTS}

\section{Demographic data}

In this study, 883 diagnostic full-night diagnostic PSG records were investigated. Based on our criteria, 88 patients with UARS and 365 patients with OSAS were identified. The demographic data of each group of patients with sleep-disordered breathing are shown in Table 1. The patients with UARS were significantly younger than the patients with OSAS ( $\mathrm{p}<0.001)$. Female patients constituted a significantly larger portion of the UARS group than the OSAS group $(\mathrm{p}<0.001)$. The UARS group had a significantly lower mean body mass index (BMI) than the OSAS group $(\mathrm{p}<0.001)$.

\section{Sleep questionnaire}

The mean ESS, AIS, and PSQI scores of the two groups are

Table 1. Demographic data of patients with UARS or OSAS

\begin{tabular}{lccc}
\hline Variables & UARS $(\mathrm{N}=88)$ & OSAS $(\mathrm{N}=365)$ & $\mathrm{p}$ \\
\hline Age (years) & $36.84 \pm 13.85$ & $49.52 \pm 11.79$ & $<0.001$ \\
BMI $\left(\mathrm{kg} / \mathrm{m}^{2}\right)$ & $23.51 \pm 3.34$ & $26.50 \pm 4.12$ & $<0.001$ \\
Male/Female $(\mathrm{N})$ & $45 / 43$ & $299 / 66$ & $<0.001$ \\
Female (\%) & 48.86 & 18.08 & \\
\hline
\end{tabular}

Compared using independent t-tests, with Levene test for equality of variances. BMI: body mass index, OSAS: obstructive sleep apnea syndrome, UARS: upper airway resistance syndrome

Table 2. Mean scores of ESS, AIS-8, AIS-5, and PSQI for UARS and OSAS groups

\begin{tabular}{lccccc}
\hline \multirow{2}{*}{ Variables } & \multicolumn{3}{c}{ Score } & & \multicolumn{2}{c}{ Multivariate analysis of variance } \\
\cline { 2 - 3 } \cline { 5 - 6 } & UARS $(\mathrm{N}=88)$ & OSAS $(\mathrm{N}=365)$ & $\mathrm{F}$ & $<0.001$ \\
ESS & $10.20 \pm 3.64$ & $6.80 \pm 4.18$ & 13.14 & $<0.001$ \\
AIS-8 & $11.22 \pm 4.50$ & $8.19 \pm 4.63$ & 12.56 & $<0.001$ \\
PSQ-5 & $6.08 \pm 3.63$ & $4.57 \pm 3.16$ & 12.69 & $<0.001$ \\
\hline
\end{tabular}

Obtained by multivariate analysis of covariance controlling for age and sex as covariates. AIS: Athens Insomnia Scale, ESS: Epworth Sleepiness Scale, OSAS: obstructive sleep apnea syndrome, PSQI: Pittsburgh Sleep Quality Index, UARS: Upper Airway Resistance Syndrome 
Table 3. Polysomnographic data of patients with UARS or OSAS

\begin{tabular}{|c|c|c|c|c|}
\hline \multirow{2}{*}{ Variables } & \multicolumn{2}{|c|}{ Score } & \multicolumn{2}{|c|}{ Multivariate analysis of variance } \\
\hline & UARS $(\mathrm{N}=88)$ & OSAS $(\mathrm{N}=365)$ & $\mathrm{F}$ & $\mathrm{p}$ \\
\hline TIB (min) & $446.66 \pm 58.53$ & $437.35 \pm 55.76$ & 1.48 & 0.22 \\
\hline TST (min) & $388.23 \pm 67.39$ & $367.46 \pm 71.95$ & 8.77 & $<0.001$ \\
\hline SE (\%) & $86.23 \pm 12.92$ & $84.65 \pm 16.05$ & 6.08 & $<0.001$ \\
\hline $\mathrm{SL}(\mathrm{min})$ & $12.00 \pm 12.49$ & $10.31 \pm 12.93$ & 6.47 & $<0.001$ \\
\hline $\mathrm{RL}(\min )$ & $110.05 \pm 71.03$ & $112.55 \pm 73.20$ & 2.77 & 0.041 \\
\hline Stage 1 (\% of TST) & $19.77 \pm 11.06$ & $33.47 \pm 16.31$ & 25.58 & $<0.001$ \\
\hline Stage 2 ( $\%$ of TST) & $50.07 \pm 11.67$ & $41.64 \pm 36.26$ & 1.70 & 0.166 \\
\hline Stage 3 ( $\%$ of TST) & $12.11 \pm 9.59$ & $10.63 \pm 27.07$ & 3.24 & 0.022 \\
\hline REM (\% of TST) & $18.03 \pm 7.26$ & $17.40 \pm 6.41$ & 0.70 & 0.552 \\
\hline Total arousal index & $19.85 \pm 9.10$ & $37.57 \pm 17.03$ & 33.62 & $<0.001$ \\
\hline AHI (n/h TST) & $2.10 \pm 2.04$ & $34.09 \pm 21.97$ & 64.80 & $<0.001$ \\
\hline FLAI (n/h TST) & $4.36 \pm 3.59$ & $4.29 \pm 5.61$ & 0.60 & 0.617 \\
\hline
\end{tabular}

Obtained by multivariate analysis of covariance controlling for age and sex as covariates. AHI: Apnea-Hypopnea Index, FLAI: Flow Limitation with Arousals Index, OSAS: obstructive sleep apnea syndrome, RL: REM latency, SE: sleep efficiency, SL: sleep latency, TIB: time in bed, TST: total sleep time, UARS: upper airway resistance syndrome

Table 4. Mean SCL-90-R scores of UARS and OSAS groups

\begin{tabular}{|c|c|c|c|c|}
\hline \multirow{2}{*}{ Variables } & \multicolumn{2}{|c|}{ Score } & \multicolumn{2}{|c|}{ Multivariate analysis of variance } \\
\hline & UARS $(\mathrm{N}=88)$ & OSAS $(\mathrm{N}=365)$ & $\mathrm{F}$ & $\mathrm{p}$ \\
\hline Somatization & $51.53 \pm 10.28$ & $48.04 \pm 8.86$ & 3.46 & 0.016 \\
\hline Obsessive-compulsiveness & $54.19 \pm 12.98$ & $44.47 \pm 8.92$ & 22.62 & $<0.001$ \\
\hline Interpersonal sensitivity & $52.34 \pm 12.92$ & $44.34 \pm 8.64$ & 21.39 & $<0.001$ \\
\hline Depression & $53.50 \pm 13.30$ & $44.43 \pm 8.63$ & 19.47 & $<0.001$ \\
\hline Anxiety & $51.06 \pm 11.47$ & $45.45 \pm 8.42$ & 8.77 & $<0.001$ \\
\hline Hostility & $51.61 \pm 12.19$ & $45.43 \pm 8.02$ & 13.81 & $<0.001$ \\
\hline Phobic anxiety & $50.24 \pm 11.59$ & $46.74 \pm 8.58$ & 7.05 & $<0.001$ \\
\hline Paranoid ideation & $49.90 \pm 12.05$ & $43.47 \pm 8.11$ & 13.35 & $<0.001$ \\
\hline Psychoticism & $51.54 \pm 11.63$ & $45.74 \pm 7.81$ & 12.09 & $<0.001$ \\
\hline GSI & $52.49 \pm 12.25$ & $44.37 \pm 8.78$ & 16.89 & $<0.001$ \\
\hline PSDI & $52.90 \pm 11.01$ & $47.52 \pm 9.33$ & 7.00 & $<0.001$ \\
\hline PST & $51.83 \pm 11.60$ & $43.85 \pm 11.09$ & 12.15 & $<0.001$ \\
\hline
\end{tabular}

Obtained by multivariate analysis of covariance controlling for age and sex as covariates. GSI: Global Severity Index, OSAS: obstructive sleep apnea syndrome, PSDI: Positive Symptom Distress Index, PST: positive symptom total, SCL-90-R: Symptom Checklist-90-Revision, UARS: upper airway resistance syndrome

presented in Table 2. The ESS scores for UARS and OSAS were 10.2 and $6.8(\mathrm{p}<0.001)$, respectively. AIS and PSQI mean scores were significantly higher in the UARS group than in the OSAS group $(\mathrm{p}<0.001)$.

\section{Polysomnographic features}

Table 3 summarizes the various PSG measures. Several polysomnographic variables were significantly different between the UARS and OSAS patients. Patients with UARS had higher mean total sleep time, sleep latency, NREM 3, and sleep efficiency. The total arousal index $(\mathrm{p}<0.001)$ was lower in
UARS patients on average than in OSAS patients $(19.85 \pm 9.10$ versus $37.57 \pm 17.03, \mathrm{p}<0.001)$.

\section{Symptom Checklist-90-Revision}

The mean SCL-90-R scores of the two groups are presented in Table 4 . The mean scores on somatization ( $\mathrm{p}=0.016)$, obsessive-compulsiveness, interpersonal sensitivity, depression, anxiety, hostility, phobic anxiety, paranoid ideation, psychoticism, global severity index, positive symptom distress index, and positive symptom total (all $\mathrm{p}<0.001$ ) for the UARS group were significantly higher than for the OSAS group. 
Table 5. Mean EPQ subscale scores of UARS and OSAS groups

\begin{tabular}{lcccrrr}
\hline \multirow{2}{*}{ Variables } & \multicolumn{3}{c}{ Score } & & \multicolumn{2}{c}{ Multivariate analysis of variance } \\
\cline { 2 - 3 } \cline { 5 - 6 } & UARS $(\mathrm{N}=88)$ & OSAS $(\mathrm{N}=365)$ & \multicolumn{1}{c}{$\mathrm{F}$} & 0.006 \\
\hline Extroversion/introversion & $9.35 \pm 5.04$ & $10.74 \pm 4.66$ & 4.19 & $<0.001$ \\
Lie & $10.06 \pm 4.48$ & $12.23 \pm 4.02$ & 46.72 & 0.002 \\
Psychoticism & $2.97 \pm 2.37$ & $2.14 \pm 1.76$ & & $<.91$ & $<0.001$ \\
Neuroticism & $16.57 \pm 4.46$ & $13.10 \pm 4.89$ & & 10.39 & \\
\hline
\end{tabular}

Obtained by multivariate analysis of covariance controlling for age and sex as covariates. EPQ: Eysenck's Personality Questionnaire, OSAS: obstructive sleep apnea syndrome, UARS: upper airway resistance syndrome

\section{Eysenck personality questionnaire}

The mean EPQ scores of the two groups are presented in Table 5. The mean score of extroversion/introversion was significantly lower in the UARS group than the OSAS group $(\mathrm{p}=0.006)$. The mean score of the lie scale for the UARS group was significantly higher than for the OSAS group $(\mathrm{p}<0.001)$. The mean score of psychoticism was significantly higher in the UARS group than the OSAS group ( $\mathrm{p}=0.002)$. The mean score of neuroticism for the UARS group was significantly higher than for the OSAS group $(\mathrm{p}<0.001)$.

\section{DISCUSSION}

To compare personality characteristics and psychiatric symptomatology of the UARS group with those of the OSAS group, we analyzed clinical features, SCL-90-R scores, and EPQ scores, controlling for age and gender. Patients with UARS were significantly younger than patients with OSAS on average, and the UARS group had a higher proportion of females than the OSAS group. These findings are consistent with previous studies of UARS patients. ${ }^{1,310}$ Patients with UARS had lower BMI than patients with OSAS, on average, which was also consistent with previous studies. ${ }^{4,5}$

Concerning insomnia, subjective quality of sleep and excessive daytime sleepiness, we found that patients with UARS scored significantly higher on average than those with OSAS on AIS, PSQI and ESS. This finding suggests that UARS patients perceive the quality of their sleep as being worse than OSAS patients do, and they are more likely to complain of insomnia and excessive daytime sleepiness.

Furthermore, comparing the subjective symptoms assessed by SCL-90-R between the two groups, UARS patients scored significantly higher on average than OSAS patients on somatization, obsessive-compulsiveness, interpersonal sensitivity, depression, anxiety, hostility, phobic anxiety, paranoid ideation, psychoticism, global severity index, positive symptoms distress index, and positive symptoms total. These results suggest that UARS patients suffer from depression, anxiety, and sensitivity. Therefore, patients with UARS are likely to complain of somatic symptoms and perceive the quality of their sleep as be- ing worse than OSAS patients.

Nevertheless, none of the PSG findings were able to explain the worse quality of sleep experienced by UARS patients compared to OSAS patients. Patients with UARS had significantly longer total sleep time and higher sleep efficiency than patients with OSAS. In addition, the UARS group had a markedly lower mean total arousal index than the OSAS group. These findings suggest that sleep quality evaluated by PSG of patients with UARS tends to be better than that of patients with OSAS.

To explain the discrepancy between objective PSG findings and subjective symptomatology, we hypothesize that UARS patients have different personality characteristics from OSAS patients. The EPQ, an instrument for assessing personality devised by Hans Jürgen Eysenck, was used for this purpose in this study. Eysenck's personality theory is based primarily on physiology and genetics. Although he considered learned habits of great importance, he considered personality differences, called temperament, as growing out of individuals' genetic inheritance. Temperament is that aspect of our personalities that is genetically based, inborn, and there from birth or even before.

Results from the EPQ temperament assessment showed that patients with UARS displayed lower scores on the extroversion scale than patients with OSAS, indicating that UARS patients tended to be introverted, pessimistic, and unsociable. UARS patients also had lower scores on the lie scale, suggesting that they tended to show their symptoms and severely complain, given that the lie scale reflects efforts toward social desirability and dissimulation. Patients with UARS scored significantly higher on the psychoticism scale, suggesting that they tended to be more solitary, troublesome, and hostile. Finally, the UARS group had a higher mean score for neuroticism than the OSAS group. Thus, patients with UARS can easily become anxious, moody, and depressed, leading to the results found with SCL-90-R in UARS patients.

In conclusion, the results of this study confirm our impression that UARS patients differ from OSAS patients in personality characteristics. Patients with UARS tend to be introverted, neurotic, and are likely to experience anxiety, sensitivity, and somatization. These findings suggest that patients in this group 
are likely to take their symptoms seriously and respond nervously. Recently, several studies have found that patients with UARS present with chronic insomnia, excessive daytime sleepiness, fatigue, and multiple somatic symptoms more frequently than do patients with OSAS. ${ }^{4,5}$ The results of the present study may explain why UARS patients complain more frequently of somatic and subjective symptoms, although patients with UARS have a less severe breathing disorder than patients with OSAS.

Guilleminault et al. ${ }^{1}$ defined UARS as the combination of a clinical complaint (daytime sleepiness) with demonstrations of flow limitation and increased respiratory efforts with arousal They suggested that UARS consists of transient, repetitive arousals that are linked to increased respiratory effort and lead to sleep fragmentation. Thus, they regarded this syndrome as sleep-disordered breathing. Our findings, however, may suggest that upper airway resistance and resulting arousals alone are not sufficient to explain this syndrome. Because UARS subjects are sensitive and neurotic in temperament, this may lead them to have frequent arousals in response to slight increases in respiratory effort. That is, UARS may not be simply sleep-disordered breathing; this syndrome may be an overlapping syndrome of comorbid psychiatric susceptibility along with increased respiratory effort.

In this study, we demonstrate that UARS differs from OSAS in the patients' personality characteristics. Strengths of this study include its relatively large sample size and the advantage that the evaluations took place at one sleep center. Moreover, this is the first in-depth study to investigate the personality characteristics of UARS patients.

There were several limitations in this study. One, we did not compare the two types of sleep-disordered breathing with normal controls. Also, the study subjects were patients who visited a university hospital sleep center for sleep-related symptoms, which may limit the generalizability of the study findings. Another limitation is that esophageal pressure (Pes) monitoring was not performed during PSG. Additionally, the EPQ results may not only reflect the premorbid personality, but may also be affected by current psychiatric symptoms. However, the EPQ was devised to evaluate the genetically based temperament. Finally, the fact that structured interviews for personality were not conducted is another limitation.

In summary, our findings suggest that patients with UARS are more likely to have neurotic personalities and tend to be more anxious and sensitive than patients with OSAS. UARS patients tend to perceive their subjective quality of sleep as being worse than OSAS patients do, although respiratory abnormality is not as severe as that observed in OSAS. Further investigations are warranted to support and expand the results of this study, preferably with larger samples.

\section{Acknowledgments}

This study was supported by the Korea Health 21 R\&D Project funded by the Ministry of Health \& Welfare, Republic of Korea (HI11C1901), the National Research Foundation of Korea (2012M3A9C7050135), and the Future Environmental R\&D grant funded by the Korea Environmental Industry and Technology Institute (No. RE201206020).

\section{REFERENCES}

1. Guilleminault C, Stoohs R, Clerk A, Cetel M, Maistros P. A cause of excessive daytime sleepiness. The upper airway resistance syndrome. Chest 1993;104:781-787.

2. Guilleminault C, Li K, Chen NH, Poyares D. Two-point palatal discrimination in patients with upper airway resistance syndrome, obstructive sleep apnea syndrome, and normal control subjects. Chest 2002;122:866-870

3. Gold AR, Marcus CL, Dipalo F, Gold MS. Upper airway collapsibility during sleep in upper airway resistance syndrome. Chest 2002;121: 1531-1540.

4. Gold AR, Dipalo F, Gold MS, O'Hearn D. The symptoms and signs of upper airway resistance syndrome: a link to the functional somatic syndromes. Chest 2003;123:87-95.

5. Stoohs RA, Knaack L, Blum HC, Janicki J, Hohenhorst W. Differences in clinical features of upper airway resistance syndrome, primary snoring, and obstructive sleep apnea/hypopnea syndrome. Sleep Med 2008; 9:121-128.

6. Guilleminault C, Kirisoglu C, Poyares D, Palombini L, Leger D, FaridMoayer M, et al. Upper airway resistance syndrome: a long-term outcome study. J Psychiatr Res 2006;40:273-279.

7. Stoohs RA, Philip P, Andries D, Finlayson EV, Guilleminault C. Reaction time performance in upper airway resistance syndrome versus obstructive sleep apnea syndrome. Sleep Med 2009;10:1000-1004.

8. Guilleminault C, Faul JL, Stoohs R. Sleep-disordered breathing and hypotension. Am J Respir Crit Care Med 2001;164:1242-1247.

9. Guilleminault C, Poyares D, Rosa A, Huang YS. Heart rate variability, sympathetic and vagal balance and EEG arousals in upper airway resistance and mild obstructive sleep apnea syndromes. Sleep Med 2005; 6:451-457.

10. Guilleminault $C$, Chowdhuri S. Upper airway resistance syndrome is a distinct syndrome. Am J Respir Crit Care Med 2000;161:1412-1413.

11. Guilleminault C, Black J, Carrillo O. EEG arousal and upper airway resistance syndrome. Electroencephalogr Clin Neurophysiol 1997;103:11.

12. Millman RP, Fogel BS, Mcnamara ME, Carlisle CC. Depression as a manifestation of obstructive sleep apnea: reversal with nasal continuous positive airway pressure. J Clin Psychiatry 1989;50:348-351.

13. Reynolds CF 3rd, Kupfer DJ, Mceachran AB, Taska LS, Sewitch DE, Coble PA. Depressive psychopathology in male sleep apneics. J Clin Psychiatry 1984;45:287-290.

14. Wells R, Carney R, Freedland K, Duntley S, Day RC. Relationship between subjective sleep quality and depression in patients with obstructive sleep apnea. Sleep 2003;26(Suppl):A258.

15. Aikens JE, Caruana-Montaldo B, Vanable PA, Tadimeti L, Mendelson WB. MMPI correlates of sleep and respiratory disturbance in obstructive sleep apnea. Sleep 1999;22:362-369.

16. Ramos-Platon MJ, Espinar-Sierra J. Changes in psychopathological symptoms in sleep apnea patients after treatment with nasal continuous positive airway pressure. Int J Neurosci 1992;62:173-195.

17. Cheshire K, Engleman H, Deary I, Shapiro C, Douglas NJ. Factors impairing daytime performance in patients with sleep apnea/hypopnea syndrome. Arch Intern Med 1992;152:538-541.

18. American Academy of Sleep Medicine. International Classification of Sleep Disorders, 2nd Edition. Westchester, IL: American Academy of Sleep Medicine; 2005.

19. American Psychiatric Association. Diagnostic and Statistical Manual of Mental Disorders, 4th Edition. Washington DC: American Psychi- 
atric Press Inc.; 1994.

20. Soldatos CR, Dikeos DG, Paparrigopoulos TJ. Athens insomnia scale: validation of an instrument based on ICD-10 criteria. J Psychosom Res 2000;48:555-560.

21. Buysse DJ, Reynolds CF 3rd, Monk TH, Berman SR, Kupfer DJ. The Pittsburgh Sleep Quality Index: a new instrument for psychiatric practice and research. Psychiatry Res 1989;28:193-213.

22. Johns MW. Reliability and factor analysis of the Epworth sleepiness scale. Sleep 1992;15:376-381.

23. Cho YW, Lee JH, Son HK, Lee SH, Shin C, Johns MW. The reliability and validity of the Korean version of the Epworth sleepiness scale. Sleep Breath 2011;15:377-384.

24. Derogatis LR, Rickels K, Rock AF. The SCL-90 and the MMPI: a step in the validation of a new self-report scale. Br J Psychiatry 1976;128: 280-289.

25. Kim KI, Kim KJ, Won HT. Korean Manual of Symptom Checklist90-Revision. Seoul: Jung Ang Juk Sung Publisher; 1984.

26. Lee HS, Eysenck HJ. The Manual of the Eysenck Personality Questionnaire. Seoul: Hakjisa; 1997.

27. Eysenck HJ, Eysenck SB. The Manual of the Eysenck Personality Questionnaire. London: Hodder and Stoughton; 1975.

28. Caruso JC, Witkiewitz K, Belcourt-Dittloff A, Gottlieb JD. Reliability of scores from the Eysenck personality questionnaire: a reliability generalization study. Educ Psychol Meas 2001;61:675-689.

29. Iber C, Ancoli-Israel S, Chesson A, Quan SF. The AASM Manual for the Scoring of Sleep and Associated Events: Rules, Terminology and Technical Specifications. Westchester, IL: American Academy of Sleep Medicine; 2007.
30. Ong KC, Clerk AA. Comparison of the severity of sleep-disordered breathing in Asian and Caucasian patients seen at a sleep disorders center. Respir Med 1998;92:843-848.

31. Li KK, Kushida C, Powell NB, Riley RW, Guilleminault C. Obstructive sleep apnea syndrome: a comparison between Far-East Asian and white men. Laryngoscope 2000;110:1689-1693.

32. Hanihara T. Comparison of craniofacial features of major human groups. Am J Phys Anthropol 1996;99:389-412.

33. Wong ML, Sandham A, Ang PK, Wong DC, Tan WC, Huggare J. Craniofacial morphology, head posture, and nasal respiratory resistance in obstructive sleep apnoea: an inter-ethnic comparison. Eur J Orthod 2005;27:91-97.

34. Liu YH, Lowe AA, Zeng XL, Fu MK, Fleetham JA. Cephalometric comparisons between Chinese and Caucasian patients with obstructive sleep apnea. Am J Orthod Dentofacial Orthop 2000;117:479-485.

35. Partinen M, Guilleminault C, Querasalva MA, Jamieson A. Obstructive sleep-apnea and cephalometric roentgenograms - the role of anatomic upper airway abnormalities in the definition of abnormal breathing during sleep. Chest 1988;93:1199-1205.

36. Kim J, In K, Kim J, You S, Kang K, Shim J, et al. Prevalence of sleep-disordered breathing in middle-aged Korean men and women. Am J Respir Crit Care Med 2004;170:1108-1113.

37. Tachibana N, Taniguchi M. Why do we continue to use Epworth sleepiness scale? Sleep Med 2007;8:541-542.

38. Zhang JN, Peng B, Zhao TT, Xiang M, Fu W, Peng Y. Modification of the Epworth sleepiness scale in central China. Qual Life Res 2011;20: 1721-1726. 